\section{Programa Saúde da Família: ampliando a cobertura para consolidar a mudança do modelo de Atenção Básica}

\section{The Family Health Program: broadening coverage to consolidate the change in the Primary Care model}

Ministério da Saúde. Secretaria de Atenção à Saúde. Departamento de Atenção Básica 1

1 Esplanada dos Ministérios, Bloco G, $7^{\circ}$ andar, sala 751. Brasília, DF, Brasil. CEP: 70.058-900. E-mail: dab@saude. gov.br. Site: www.saude.gov.br

\section{Contexto histórico da Atenção Básica e o Programa Saúde da Família}

Nos últimos dez anos, é inegável que o Sistema Único de Saúde (SUS) passou por transformações importantes comparáveis aos vários momentos de modernização dos setores produtivos no Brasil. Se quisermos utilizar apenas uma palavra para caracterizar estas mudanças, poderíamos apontar a palavra inclusão. Ela permite definir a ampliação do acesso da população aos serviços de saúde quando, a partir da reformulação do arcabouço constitucional, ficam inscritos os princípios de universalidade, eqüidade e integralidade das ações, o que garante a qualquer cidadão, em território nacional, ser atendido pela rede de serviços públicos de saúde.

Tal fato - contrariamente ao modelo anterior, de cunho claramente excludente, centrado na doença e que privilegiava uma medicina de alto custo - posiciona o Brasil ao lado dos países que pautam seus sistemas de saúde em valores de solidariedade e cidadania. Entretanto, entre a modernidade da lei e a efetiva implantação do SUS, num país de proporções continentais, como o nosso, muitos desafios tiveram, e ainda têm que ser enfrentados. Do período (1988) que vai da aprovação da nova Constituição até os dias de hoje, dois grandes eixos se apresentam como questões complexas: a descentralização político-administrativa e a organização da atenção à saúde.

Para fazer frente a esses dois eixos, a partir de 1995, desenvolveu-se no setor um movimento denominado de "reforma da reforma" do sistema que, além das determinações legais, buscou modificar o desenho e as operações da política de saúde no sentido de acelerar o processo de descentralização, reestruturar os mecanismos de financiamento das ações de saúde e, principalmente, fortalecer a atenção básica.

Os instrumentos importantes deste movimento foram as Normas Operacionais editadas pelo Ministério da Saúde. Neste contexto, vale ressaltar a relevância da Norma Operacional Básica 01/96 que, a partir de 1998, gerou mudanças importantes relacionadas à estruturação da atenção básica, à ampliação e organização da rede de serviços públicos em várias regiões do país, que serão detalhadas adiante. Ela também foi responsável pelo aumento do volume de transferências financeiras diretas do Fundo Nacional para os Fundos Estaduais e Municipais de Saúde e pelo fortalecimento dos gestores locais. No que diz respeito ao fortalecimento da atenção básica, o avanço caracterizou-se pelo incentivo às ações consideradas estratégicas, dentre as quais a expansão do Programa de Saúde da Família (PSF).

A origem do Programa de Saúde da Família está na decisão do Ministério da Saúde, em 1991, de implantar o Programa de Agentes Comunitários de Saúde (PACS), como medida de enfrentamento dos graves índices de morbimortalidade materna e infantil na Região Nordeste do país. O PACS pode ser considerado o antecessor do PSF por alguns de seus elementos que tiveram um papel central na construção do novo programa. Entre elas, enumeramos o enfoque na família e não no indivíduo e o agir preventivo sobre a demanda, constituindo-se num instrumento de organização da mesma. De certo modo, o que se percebeu é que houve a adoção de uma prática não reducionista sobre a saúde, evitando ter como eixo apenas a intervenção médica e buscando a integração de fato com a comunidade.

$\mathrm{O}$ reconhecimento da crise do modelo anterior suscitou a necessidade emergencial de uma nova estratégia estruturante, contemplando a incorporação de recursos humanos e tecnologias contextualizadas 
nas novas práticas. Com esse propósito, iniciou-se a formulação das diretrizes do Programa Saúde da Família, lançado em março de 1994. Já à época, ele foi apresentado como a estratégia capaz de provocar mudanças no modelo assistencial ao romper com o comportamento passivo das unidades básicas de saúde e estender suas ações para e junto à comunidade.

As unidades do Saúde da Família passariam a atuar com equipes multiprofissionais, compostas, minimamente, por um médico, uma enfermeira, um ou dois auxiliares de enfermagem e de quatro a seis agentes comunitários. Assim, se assumia o desafio do trabalho em equipe multidisciplinar, com responsabilização sobre um território onde vivem, em média, mil famílias. As definições de responsabilidade territorial e de adscrição de famílias (introduzidas no PACS e ampliadas no PSF) conferiam ao programa uma característica especial: a potencialidade para resgatar os vínculos de compromisso e de co-responsabilidade entre os serviços de saúde, os profissionais e a população.

O ideário do Programa de Saúde da Família, calcado nos princípios da Reforma Sanitária, assume como foco a reorganização da atenção básica, garantindo a oferta de serviços à população brasileira e o fortalecimento dos princípios da universalidade, acessibilidade, integralidade e eqüidade do SUS. Além disso, privilegia outras interfaces do sistema em diversos sentidos: por sua vertente municipalista, busca estimular a organização dos sistemas locais; pauta-se a partir da aproximação dos serviços com sua própria realidade; e envolve os atores sociais destas mesmas realidades. Assim, o controle social e a participação popular se fortaleceram à medida que o programa se ampliou.

O conceito de saúde, que permeia todo o desenvolvimento do PSF, influiu de forma decisiva na mudança da organização do processo de trabalho. A equipe do Saúde da Família assumia, assim, o desafio da atenção continuada, resolutiva e pautada pelos princípios da promoção da saúde, nos quais a ação intersetorial era considerada essencial para a melhoria dos indicadores de saúde e de qualidade de vida da população acompanhada.

Ao PSF foi, então, atribuída a função de desenvolver ações básicas, no primeiro nível de atenção à saúde propondo-se a uma tarefa maior do que a simples extensão de cobertura e ampliação do acesso. $\mathrm{O}$ programa deveria promover a reorganização da prática assistencial com novos critérios de abordagem, provocando reflexos em todos os níveis do sistema. Daí seu potencial estruturante sobre o modelo, o que justificou a decisão do Ministério da Saúde, nos anos seguintes, de considerá-lo como a principal estratégia de qualificação da atenção básica e reformulação do modelo assistencial.

O PSF não encontrou, no início de sua implantação, um cenário favorável ao seu fortalecimento. $\mathrm{O}$ processo de formulação não estava totalmente concluído e as condições necessárias à sua sustentabilidade não foram definidas de modo suficiente no âmbito institucional. Não havia, portanto, definições quanto ao seu financiamento (que era viabilizado por meio de convênios) e nem quanto à política de recursos humanos, pilares fundamentais para o seu desenvolvimento. Além dessas restrições, havia a própria resistência à implantação de uma proposta que confrontava as formas tradicionais de organização dos serviços.

Em 1995, o PACS e o PSF que estavam sob responsabilidade da Coordenação de Saúde da Comunidade do Departamento de Operações, da Fundação Nacional de Saúde, foram transferidos para a Secretaria de Assistência à Saúde (SAS), do Ministério da Saúde.

Esses dois programas foram incluídos nas regras de financiamento da assistência, vigentes à época. Os repasses baseavam-se na produção de serviços, limitados por um teto financeiro que, nem sempre, permitia o atendimento às reais necessidades da população. Se, por um lado, essa forma de financiamento conferiu regularidade maior à transferência dos recursos federais aos municípios que implementaram estes programas, por outro lado, como os recursos não estavam vinculados obrigatoriamente às ações dos programas ou mesmo à atenção básica, eram, em geral, alocados em serviços ou ações que demandavam mais recursos. Conseqüentemente, era comum faltarem recursos para a implementação do PSF e do PACS. Considerava-se que, na prática, a lógica de alocação de recursos acabava priorizando a doença e não a saúde.

As contradições geradas pelo PSF fortaleceram o debate em torno das modalidades de financiamento do setor, o que se refletiu na formulação da Norma Operacional Básica de 1996 (NOB/SUS/96). Foi então, criado o Piso de Atenção Básica (PAB), pelo qual todo município teria um valor específico repassado pelo Fundo Nacional de Saúde ao Fundo Municipal de Saúde, calculado com base no número de habitantes (valor per capita), para viabilizar a atenção básica de saúde a toda a população. O PAB apresentava também uma composição de recursos variáveis, sob forma de incentivos para ações e projetos prioritários, com destaque para o PACS e o PSF.

A implantação do PAB ocorreu, de fato, em 
fevereiro de 1998, quando se iniciou a transferência mensal para os municípios habilitados pela NOB/96.

A NOB/SUS/96 fortaleceu significativamente a atenção básica ao definir dois pontos fundamentais: as responsabilidades dos gestores municipais nesse nível de complexidade do sistema e o PSF como estratégia prioritária para mudança do modelo assistencial. Com a implantação do PAB (fixo e dos incentivos), o Saúde da Família passou a ter orçamento próprio e foi incluído no Plano Plurianual (PPA) do Governo Federal, em 1998.

No ano seguinte, 1999, destacaram-se, entre várias ações de importância, a elaboração e a publicação do "Manual para Organização da Atenção Básica no SUS" e o início da implementação do Pacto de Indicadores da Atenção Básica. O "Manual" foi um marco importante porque definiu as responsabilidades dos municípios nesse nível de atenção; orientou sobre repasse, aplicação e mecanismos de acompanhamento e controle dos recursos financeiros; e apontou estratégias prioritárias para organização da atenção básica, com destaque ao Programa de Saúde da Família.

Em continuidade ao processo de definição de responsabilidades inscritas no "Manual" - como afirma o Relatório de Gestão da Secretaria de Assistência à Saúde: 1998/2001 - a implantação do Pacto foi de suma importância para articular, em torno de um mesmo objetivo, as práticas e percepções das diferentes áreas técnicas das secretarias estaduais e municipais de saúde e do próprio Ministério. O Pacto imprimiu maior visibilidade às ações desenvolvidas pelos municípios habilitados na condição de Gestão Plena da Atenção Básica (definida na NOB/SUS/96). Ele possibilitou o acompanhamento das atividades, no âmbito da rede de serviços, e também a avaliação do seu impacto sobre as condições de saúde da população. O relatório afirma ainda que a implementação do Pacto colocou, de modo objetivo, compromissos a serem assumidos, de forma acordada pelos gestores municipais, em substituição à prestação de contas contábil da utilização dos recursos do PAB.

Com relação aos recursos humanos, a consolidação do PSF estava condicionada à existência, nos serviços, de profissionais com conhecimentos, atitudes e habilidades adequados ao novo modelo, capazes de uma prática multiprofissional e interdisciplinar, preparados para este novo processo de trabalho. No entanto, era e é preciso reconhecer que, no Brasil, o ensino das profissões de saúde ainda está baseado no modelo flexneriano e fragmentado, que usa a lógica da especialização na produção do trabalho. Assim, era necessário para implementar a atenção básica, repensar a questão para enfrentar esse desafio. Em 1997, foram propostos, no Ministério da Saúde, os Pólos de Capacitação, Formação e Educação Permanente de Recursos Humanos para o Programa Saúde da Família. Trata-se de espaços de articulação entre serviços e instituições de ensino superior com a missão de implementar processos de capacitação destinados aos profissionais do Programa.

Podemos afirmar, portanto, que o ano de 1998 foi um marco no processo de consolidação do PSF, iniciativa definida pelo Ministério da Saúde como "estratégia estruturante para a organização do sistema de saúde". Para que o Sistema Único de Saúde funcionasse, o Ministério considerava que era necessária sua estruturação numa base municipal sólida e que o mecanismo para isso era a estratégia do PSF organizando a atenção básica. As ações precisavam de fortalecimento para atingir, com qualidade e sustentabilidade, a meta de 150 mil agentes comunitários de saúde e 20 mil equipes do Saúde da Família em atuação no país, até o fim de 2002.

Do ponto de vista do fortalecimento institucional-administrativo para a atenção básica, em 1999, ainda na Secretaria de Assistência à Saúde, foi criada a Coordenação de Atenção Básica que seria em seguida, transformada em Departamento, com estrutura de três coordenações: a primeira com a tarefa de acompanhar a implantação das equipes do Saúde da Família e o desenvolvimento das responsabilidades da atenção básica; a segunda com a tarefa de executar os projetos de qualificação do processo de trabalho da atenção básica/PSF, principalmente na área de capacitação dos profissionais de saúde; e a terceira com a função de acompanhar e avaliar o processo de reorganização da atenção básica, com ênfase na estratégia do PSF.

Em fevereiro de 2000, o Departamento de Atenção Básica foi formalizado na estrutura do Ministério da Saúde. Desde então, passou a ser subordinado à Secretaria de Políticas de Saúde (SPS), que foi reformulada para assumir a missão de agregar os esforços das diversas áreas técnicas e de intervenção programática para fortalecimento da atenção básica.

Outro aspecto que merece relevância para o fortalecimento da atenção básica refere-se à publicação da Norma Operacional da Assistência do SUS (NOAS/SUS), em janeiro de 2001, com o objetivo geral de "promover maior equidade na alocação de recursos e no acesso da população às ações e serviços de saúde em todos os níveis da atenção".

A NOAS trata da organização dos serviços municipais no contexto da micro e macro-regionalização, oferecendo estratégias intermunicipais para a organização dos serviços de atenção básica, orien- 
tando a construção das redes regionalizadas e hierarquizadas de serviços de saúde de qualidade e resolutivas. Aponta três eixos que, de forma articulada, visam contribuir para a organização do sistema:

- Elaboração de um Plano Diretor de Regionalização coordenado pelas Secretarias Estaduais de Saúde, envolvendo o conjunto dos municípios como forma de organização de sistemas funcionais e resolutivos em seus diversos níveis;

- Fortalecimento da capacidade gestora do SUS voltada para consolidação do caráter público da gestão do sistema; e

- Atualização dos critérios e do processo de habilitação de estados e municípios, passando do caráter meramente cartorial para um processo de responsabilização real, com pactuação de compromissos e metas pelos gestores.

Nesse contexto, o Departamento de Atenção Básica, na perspectiva do fortalecimento deste nível de atenção, passa a participar ativamente do acompanhamento da implantação da NOAS, nos estados e municípios, através de sua Coordenação de Gestão, que atua em parceria com o Departamento de Descentralização da Gestão da Assistência da Secretaria de Assistência à Saúde do Ministério da Saúde (SAS/MS)

Muito se tem investido no processo de reflexão, avaliação e reorganização da atenção básica. Toda a experiência adquirida foi refletida e incorporada na NOAS, visando ampliar as condições para a mudança do modelo assistencial.

\section{Atenção Básica na Norma Operacional de Assistência à Saúde (NOAS/SUS)}

A NOAS/SUS 01/2001 define as responsabilidades e ações estratégicas mínimas que todos os municípios brasileiros devem desenvolver no âmbito da Atenção Básica. São elas: controle da Tuberculose, eliminação da Hanseníase, controle da Hipertensão, controle da Diabetes Melittus, ações de Saúde Bucal, ações de Saúde da Criança e ações de Saúde da Mulher.

Além dessas ações, os estados e municípios podem definir, baseados em seu perfil demográfico e epidemiológico e suas prioridades políticas, outras ações que julguem estratégicas e necessárias.

Busca-se articular a atenção básica com a rede de serviços de maior complexidade como parte indissolúvel dessa e como porta de entrada do sistema. Esta articulação encontra-se materializada através de instrumentos específicos, a saber:

- Programação Pactuada Integrada (PPI) que define ações de saúde a serem executadas localmente, entre estados e municípios, com as respectivas responsabilidades físicas e orçamentárias em cada nível de atenção, visando a garantia do acesso da população a todos os serviços de saúde;

- Plano Diretor de Regionalização (PDR), elaborado pelos estados, que agrupa seus municípios em níveis crescentes de complexidade para utilização racional dos serviços de saúde;

- Plano Diretor de Investimentos (PDI), documento estadual, que define a aplicação de recursos financeiros na adequação da rede assistencial às ações de saúde pactuadas, inclusive contemplando a necessidade de instalação de serviços de atenção básica onde ainda não houver suficiência dos mesmos.

A riqueza do processo de discussão e implantação da NOAS torna-se também um momento de reflexão e avaliação da atenção básica e de todo o potencial existente nesse nível, para a efetiva mudança do modelo de atenção à saúde proposto pelo SUS.

Responsabilidades e ações estratégicas mínimas da Atenção Básica: 


\begin{tabular}{|c|c|}
\hline \multicolumn{2}{|r|}{ Controle da tuberculose } \\
\hline Responsabilidades & Atividades \\
\hline Busca ativa de casos & Identificação de sintomáticos respiratórios (SR) \\
\hline Diagnóstico clínico de casos & Exame clínico de SR e comunicantes \\
\hline Acesso a exames para diagnósti- & Realização ou referência para Baciloscopia \\
\hline $\begin{array}{l}\text { co e controle: laboratorial e } \\
\text { radiológico }\end{array}$ & $\begin{array}{l}\text { Realização ou referência para exame radiológico em SR com baciloscopias } \\
\text { negativas (BK -) }\end{array}$ \\
\hline Cadastramento dos portadores & Alimentação e análise dos sistemas de informação \\
\hline Tratamento dos casosBK+ (super- & Tratamento supervisionado dos casos BK+ \\
\hline visionado) e & Tratamento auto-administrado dos casos BK - \\
\hline \multirow[t]{3}{*}{ BK (auto-administrado) } & Fornecimento de medicamentos \\
\hline & Atendimentos às intercorrências \\
\hline & Busca de faltosos \\
\hline \multirow[t]{3}{*}{ Medidas preventivas } & Vacinação com BCG \\
\hline & Pesquisa de comunicantes quimioprofilaxia \\
\hline & Ações educativas \\
\hline
\end{tabular}

\section{Quadro 2}

Hanseníase

\begin{tabular}{|c|c|}
\hline \multicolumn{2}{|r|}{ Eliminação da Hanseníase } \\
\hline Responsabilidades & Atividades \\
\hline Busca ativa de casos & Identificação de sintomáticos dermatológicos entre usuários \\
\hline \multirow[t]{2}{*}{ Diagnóstico clínico de casos } & Exame de sintomáticos dermatológicos e comunicantes de casos \\
\hline & Classificação clínica dos casos (multibacilares e paucibacilares) \\
\hline Cadastramento dos portadores & Alimentação e análise dos sistemas de informação \\
\hline Tratamento supervisionado dos & Acompanhamento ambulatorial e domiciliar \\
\hline \multirow[t]{4}{*}{ casos } & Avaliação dermato-neurológica \\
\hline & Fornecimento de medicamentos \\
\hline & Curativos \\
\hline & Atendimento de intercorrências \\
\hline Controle das incapacidades físi- & Avaliação e classificação das incapacidades físicas \\
\hline \multirow[t]{2}{*}{ cas } & Aplicação de técnicas simples de prevenção e tratamento de incapacidades \\
\hline & Atividades educativas \\
\hline \multirow[t]{4}{*}{ Medidas preventivas } & Pesquisa de comunicantes \\
\hline & Divulgação de sinais e sintomas da hanseníase \\
\hline & Prevenção de incapacidades físicas \\
\hline & Atividades educativas \\
\hline
\end{tabular}


Hipertensão

\begin{tabular}{|c|c|}
\hline \multicolumn{2}{|r|}{ Controle da hipertensão } \\
\hline Responsabilidades & Atividades \\
\hline Diagnóstico de casos & Diagnóstico clínico \\
\hline Cadastramento dos portadores & Alimentação e análise dos sistemas de informação \\
\hline Busca ativa de casos & Medição de P.A. de usuários, Visita domiciliar \\
\hline Tratamento dos casos & $\begin{array}{l}\text { Acompanhamento ambulatorial e domiciliar. Fornecimento de medicamentos } \\
\text { Acompanhamento domiciliar de pacientes com seqüelas de acidente vas- } \\
\text { cular cerebral (AVC) e outras complicações }\end{array}$ \\
\hline $\begin{array}{l}\text { Diagnóstico precoce de compli- } \\
\text { cações }\end{array}$ & $\begin{array}{l}\text { Realização ou referência para exames laboratoriais complementares } \\
\text { Realização ou referência para ECG. Realização ou referência para RX de tórax }\end{array}$ \\
\hline $\begin{array}{l}\text { Primeiro atendimento de urgên- } \\
\text { cia }\end{array}$ & $\begin{array}{l}\text { Primeiro atendimento às crises hipertensivas e outras complicações } \\
\text { Acompanhamento domiciliar. Fornecimento de medicamentos }\end{array}$ \\
\hline Medidas preventivas & $\begin{array}{l}\text { Ações educativas para controle de condições de risco (obesidade, vida } \\
\text { sedentária, tabagismo) e prevenção de complicações }\end{array}$ \\
\hline
\end{tabular}

Quadro 4

Diabetes Melittus

\section{Controle da diabetes Melittus}

Responsabilidades

Diagnóstico de casos

Cadastramento dos portadores

Busca ativa de casos

Tratamento dos casos

Monitoração dos níveis de glicose do paciente

Diagnóstico precoce de complicações

Primeiro atendimento de urgência

Encaminhamento de casos graves para outro nível de complexidade

Medidas preventivas e de promoção da saúde
Atividades

Investigação em usuários com fatores de risco

Alimentação e análise de sistemas de informação

Visita domiciliar

Acompanhamento ambulatorial e domiciliar

Educação terapêutica em diabetes

Fornecimento de medicamentos. Curativos

Realização de exame dos níveis de glicose (glicemia capilar) pelas unidades de saúde

Realização ou referência laboratorial para apoio ao diagnóstico de complicações

Realização ou referência para ECG

Primeiro atendimento às complicações agudas e outras intercorrências Acompanhamento domiciliar

Agendamento do atendimento

Ações educativas sobre condições de risco (obesidade, vida sedentária) Ações educativas para prevenção de complicações (cuidados com os pés, orientação nutricional, cessação do tabagismo e alcoolismo; controle da P.A e das dislipidemias). Ações educativas para auto-aplicação de insulina 
Saúde bucal

\begin{tabular}{|ll|}
\hline & \multicolumn{1}{c}{ Açães de saúde bucal } \\
\hline $\begin{array}{l}\text { Responsabilidades } \\
\text { Prevenção dos problemas odon- } \\
\text { tológicos, prioritariamente, na } \\
\text { população de } 0 \text { a } 14 \text { anos e ges- }\end{array}$ & $\begin{array}{l}\text { Atividades } \\
\text { Procedimentos coletivos: levantamento epidemiológico; escovação super- } \\
\text { visionada e evidenciação de placa; bochechos com flúor; educação em } \\
\text { santes }\end{array}$ \\
$\begin{array}{l}\text { Cadastramento bucal } \\
\text { Tratamento dos problemas } \\
\text { odontológicos, prioritariamente, } \\
\text { na população de } 0 \text { a } 14 \text { anos e }\end{array}$ & Alimentação e análise de sistemas de informação \\
gestantes & Consulta e outros procedimentos odontológicos \\
Atendimento a urgências odon- & Consulta não agendada \\
tológicas &
\end{tabular}

Quadro 6

Saúde da criança

\begin{tabular}{|ll|}
\hline Responsabilidades & Ações de Saúde da Criança \\
\hline Vigilância nutricional & Atividades \\
& Acompanhamento do crescimento e desenvolvimento \\
& Promoção do aleitamento materno \\
& Realização ou referência para exames laboratoriais \\
& Combate às carências nutricionais \\
& Implantação e alimentação regular do SISVAN??? \\
& Realização do esquema vacinal básico de rotina \\
& Busca de faltosos \\
& Realização de campanhas e intensificações \\
& Alimentação e acompanhamento dos sistemas de informação \\
Imunização & Assistência às Infecçães Respiratórias Agudas (IRA) em menores de cinco \\
& anos Assistência às doenças diarréicas em crianças menores de cinco anos \\
Assistências às doenças preva- & Assistência a outras doenças prevalentesAtividades educativas de pro- \\
lentes noção da saúde e prevenção das doençasGarantia de acesso a referência & hospitalar e ambulatorial especializada, quando necessário (programada \\
e negociada, com mecanismos de regulação)Realização ou referência para & exames laboratoriais \\
\hline
\end{tabular}


Saúde da Mulher

\begin{tabular}{|c|c|}
\hline & Ações de Saúde da Mulher \\
\hline $\begin{array}{l}\text { Responsabilidades } \\
\text { Pré-natal }\end{array}$ & $\begin{array}{l}\text { Atividades } \\
\text { Diagnóstico de gravidez } \\
\text { Cadastramento de gestantes no primeiro trimestre } \\
\text { Classificação de risco gestacional desde a primeira consulta } \\
\text { Suplementação alimentar para gestantes com baixo peso } \\
\text { Acompanhamento de pré-natal de baixo risco } \\
\text { Vacinação anti-tetânica } \\
\text { Avaliação do puerpério } \\
\text { Realização ou referência para exames laboratoriais de rotina } \\
\text { Alimentação e análise de sistemas de Informação } \\
\text { Atividades educativas para promoção da saúde }\end{array}$ \\
\hline $\begin{array}{l}\text { Prevenção de câncer de colo de } \\
\text { útero }\end{array}$ & $\begin{array}{l}\text { Rastreamento de câncer de colo de útero } \\
\text { Coleta de material para exame de citopatologia } \\
\text { Realização ou referência para exame citopatológico } \\
\text { Alimentação dos sistemas de informação }\end{array}$ \\
\hline Planejamento familiar & $\begin{array}{l}\text { Consulta médica e de enfermagem } \\
\text { Fornecimento de medicamentos e de métodos anticoncepcionais } \\
\text { Realização ou referência para exames laboratoriais }\end{array}$ \\
\hline
\end{tabular}

\section{Acompanhamento da implantação da Norma Operacional de Assistência à Saúde nos estados e municípios}

A coordenação de gestão do Departamento de Atenção Básica (DAB) vem acompanhando estados e municípios na implantação da NOAS com o objetivo de potencializar a oportunidade de valorização e inserção sistêmica da $\mathrm{AB}$, e conseqüentemente do PSF, na rede de serviços.

Esse trabalho tomou um caráter de parceria e cooperação efetiva na maioria dos estados. Por um lado, permitiu a disseminação de boas experiências entre as secretarias estaduais. Por outro, o trabalho do DAB pôde ser aprimorado porque sua equipe teve a oportunidade de compartilhar com os técnicos estaduais e municipais os desafios da operacionalização da própria NOAS.

$O$ advento da NOAS contribuiu significativamente para que a atenção básica assumisse papel relevante no planejamento de saúde. Entre estas contribuições citamos:

- A criação do Piso de Atenção Básica Ampliado (PAB-A), que passa a definir o valor de
$\mathrm{R} \$ 10,50$ per capita/ano como o montante mínimo do recurso fixo fundo-a-fundo para a atenção primária. A habilitação dos municípios em qualquer forma de gestão preconizada pela NOAS os qualificam a receber este recurso;

- A adoção do cumprimento de critérios da atenção básica como condição para as habilitações segundo a NOAS;

- A incorporação de novos procedimentos ao elenco da atenção básica (ex: eletrocardiografia), conforme anexo dois da NOAS;

- A inserção do módulo de atenção básica no instrumento eletrônico da PPI, conforme idealizado no DAB;

- A necessidade de explicitação das ações e recursos a serem direcionados para a atenção básica nos instrumentos de programação dos estados (PDR, PPI, PDI).

Em alguns estados, a participação da atenção básica no processo de planejamento encontrou-se prejudicada devido à frágil inserção da gestão da atenção básica na estrutura do nível central das Secretarias Estaduais de Saúde (SES). 


\section{A Programação Pactuada Integrada (PPI) da Atenção Básica}

A PPI foi regulamentada pela portaria do GM/MS n ${ }^{\circ}$ 483, de 6 de abril de 2001, complementar à NOAS 01/2001. A proposta de uma programação pactuada e integrada já constava da NOB/96, mas efetivamente não havia sido implantada, exceto por experiências pontuais em alguns estados. Visando a operacionalização da PPI como um instrumento nacional, o Ministério da Saúde desenvolveu e disponibilizou um software que contemplava vários aspectos do processo de programação, iniciando pela Agenda de Saúde e Pacto da Atenção Básica e chegando à programação das ações na atenção básica, média e alta complexidade ambulatorial, programação hospitalar e do tratamento fora do domicílio, entre outros componentes. A Programação da Atenção Básica, além de compor o escopo global da PPI, possui especificidades que buscam apontar novas diretrizes para o processo de programação.

A construção desse instrumento iniciou-se em 2001, a partir da idéia de que era necessário avançar na elaboração de uma programação que não se pautasse exclusivamente na série histórica de produção. Propunha-se que incorporasse, no mínimo, as necessidades normativas apontadas pelas áreas técnicas para a atenção básica, bem como o que seria necessário e suficiente de suporte da média complexidade para atender aos estrangulamentos de serviços de referência. Ao ser incluído na NOAS, o conceito de suficiência suscitou discussões para sua definição. Para atenção básica significava definir a resolutividade das equipes e o que deveria ser encaminhado a outros níveis do sistema. Um recorte inicial para essa definição já era apontado pela própria norma ao definir as sete áreas estratégicas de atuação.

A proposta evoluiu inicialmente para inclusão de coberturas por áreas programáticas, considerando que para algumas áreas, como vacinação, era necessária a utilização da base total da população, enquanto para outras áreas, a cobertura poderia variar de acordo com a prioridade dos gestores. Após essa decisão ficou patente a necessidade de definição da prevalência de alguns agravos.

Também nesse processo foi construída uma planilha-resumo que calculava automaticamente o total de consultas médicas, procedimentos médicos básicos, consultas de enfermagem, ações do auxiliar de enfermagem, reuniões de grupo, visitas do agente comunitário, entre outros. Esse rol de ações deveria ser compatível com o que uma equipe de PSF teria que fazer para a uma população média de 3.450 habi- tantes. A totalização da programação demonstrou nas simulações cerca de 1,4 a 1,6 consultas médicas básicas hab/ano por município, o que significava entre 400 e 500 consultas por medico/equipe /mês no PSF.

Outra planilha-resumo sugerida e incorporada calculava a necessidade de exames de laboratório e ultra-sonografia obstétrica para o cumprimento dos protocolos de atendimento de pré-natal, hipertensão e diabetes. Para esse cálculo, foram utilizados os protocolos do "Manual para Organização de Laboratórios Clínicos" produzido pela Secretaria de Assistência à Saúde.

A primeira versão eletrônica foi disponibilizada a partir de maio de 2001 para os estados. Representou um esforço conjunto do DAB/SPS e do Departamento de Descentralização e Gestão da Assistência (DDGA/SAS) no sentido de subsidiar estados e municípios na elaboração da PPI conforme dito anteriormente. A programação da atenção básica, responsabilidade do DAB/SPS, desenvolveu-se tendo como pressupostos que:

- A efetiva hierarquização e regionalização do Sistema Único de Saúde dependem em grande parte da reorganização da atenção básica;

- A ampliação do acesso e da resolutividade da atenção básica é condição sine qua non para reorientação e redimensionamento dos fluxos de referência para outros níveis de atenção, facilitando o acesso da população a ações e serviços o mais próximo possível de seu domicílio;

- A reorientação dos fluxos de usuários deverá resultar na diminuição da pressão de demandas inadequadas sobre serviços de maior complexidade, liberando-os para absorver melhor as demandas para as quais de fato foram constituídos;

- A programação em saúde deve ser entendida como uma etapa do processo de planejamento, que visa a operacionalização das ações necessárias para alcançar as metas priorizadas;

- A programação da atenção básica deve subsidiar a reorganização desse nível de atenção considerando as responsabilidades e ações estratégicas mínimas definidas na NOAS/SUS/01/2001;

- As ações prioritárias devem ser definidas a partir análise da situação de saúde e dos pactos firmados (Agenda Nacional de Prioridades e Pacto da Atenção Básica), visando obter impacto sobre os indicadores de saúde da população;

- A programação não deve se limitar a dados de séries históricas de produção de procedimentos, que reproduzem exclusivamente a lógica da oferta em detrimento das necessidades de saúde da população;

- Os parâmetros assistenciais baseados em 
necessidades de serviços devem ser entendidos como referências quantitativas, que podem subsidiar os municípios na programação das ações de saúde.

Cada município deve definir a sua programação com base no perfil demográfico e epidemiológico de sua população, nas características de seu modelo assistencial e nos recursos disponíveis (instalações físicas, equipamentos, recursos humanos e possibilidades de financiamento e investimentos), levando sempre em consideração os pactos já firmados (Agenda Nacional de Prioridades e Pacto da Atenção Básica).

O processo de Programação da Atenção Básica tinha como objetivos, além da definição das necessidades de referência para outros níveis de assistência:

- A tradução operacional dos Planos Municipais de Saúde e das ações que devem ser desenvolvidas no primeiro nível de assistência para alcançar as metas assistenciais do Pacto da Atenção Básica e da Agenda Nacional de Prioridades;

- A potencialização do processo de reorganização da atenção básica e reformulação do modelo assistencial visando a humanização da assistência e a ampliação do acesso, da qualidade e da resolutividade da Atenção Básica;

- A ampliação da resolutividade da Atenção Básica, pelo acréscimo de novos procedimentos ao elenco atual do PAB, pela redefinição das responsabilidades desse nível e pela otimização da produção de serviços;

- A redefinição das necessidades de custeio e prioridades de investimento dos três níveis de governo na rede básica de saúde.

A partir desse cenário, buscou-se elaborar uma proposta de parâmetros, como subsídio à programação da atenção básica, norteada pela lógica das necessidades de serviços para a população.

Para construção dessa proposta levou-se em conta a cobertura e concentrações ideais segundo normas técnicas do Ministério da Saúde, consensos de especialistas, publicações de organismos internacionais, programações já realizadas por secretarias estaduais e municipais nos últimos anos e estudos de série histórica nacional de produção de procedimentos para os itens de urgência básica, no ano 2000, orientado pelas áreas técnicas específicas.

O detalhamento da proposta permite uma programação mais minuciosa dos serviços destacando-se, em cada um dos ciclos de vida e agravos principais, suas prioridades, reservando-se uma parte das ações à demanda espontânea.

Para a ampliação da resolutividade da atenção básica também foram consideradas ações relacionadas à urgência clínica básica, às pequenas cirurgias ambulatoriais, às ações de odontologia, entre outras, que compõem o elenco de procedimentos do PAB-A.

A proposta buscou a flexibilização de todos os parâmetros propostos para que cada município faça as adaptações necessárias ao perfil etário de sua população e à realidade epidemiológica do território municipal.

Como sugestão para o processo de programação foram apontadas questões que deveriam ser observadas pelos gestores:

- Levantamento do cadastro atualizado das unidades assistenciais básicas, para subsidiar a avaliação da possibilidade de oferta de serviços no município: o cadastro atualizado de todas as unidades e dos profissionais permite avaliar a capacidade instalada calculando os parâmetros de rendimento de profissionais, unidades e equipamentos;

- Levantamento dos dados populacionais do município, por faixa etária e gênero, para fins de cálculo das coberturas assistenciais;

- Os dados populacionais por gênero e faixa etária permitirão os cálculos de cobertura das ações para cada ciclo de vida, e ações de importância epidemiológica;

- Definição pelo município dos parâmetros de cobertura para programação das ações e serviços de saúde correspondentes aos diversos grupos e subgrupos de ações finalísticas da atenção básica que deverão levar em conta o pacto da atenção básica, acordado pelo município com a SES, as ações e áreas estratégicas constantes na NOAS, e a sugestão de parâmetros elaborada.

Dependendo da composição etária ou perfil epidemiológico do município, poderão ser priorizadas atividades ou grupos populacionais, deslocando-se metas de determinados ciclos de vida ou agravos para outros:

- Levantamento pelo município do potencial de produção dos serviços públicos e privados contratados ou conveniados existentes no seu território para os procedimentos do PAB-A;

- O município deverá avaliar a capacidade instalada em seu território para produção dos procedimentos elencados no PAB-A, já que os mesmos não deverão ser referenciados para outros municípios;

- Cálculo dos possíveis déficits de cobertura existentes, comparando a necessidade de produção para sua população e o potencial de produção dos serviços disponíveis em seu território, para que se proceda a instalação de serviços e cumprimento das obrigações em relação à produção do elenco de procedimentos do PAB-A no território municipal, visan- 
do a atenção à saúde da população.

A impossibilidade do município de assegurar a atenção básica ampliada à sua população desencadeará um planejamento de investimento para instalação de novos serviços ou otimização dos existentes, visando a garantia da atenção à saúde da população, que poderá ser remetido ao Plano Diretor de Investimentos (PDI) sob coordenação da SES.

\section{Habilitação de estados e municípios}

Todos os processos de habilitação de estados e municípios, segundo a Norma Operacional de Assistência à Saúde, precisam demonstrar, em seu conteúdo, o cumprimento dos requisitos de atenção básica, conforme explicitados e detalhados na portaria $n^{\circ}$ 2.215, de 04/12/2001. É perceptível o reforço da importância da Atenção Básica em relação à agenda política dos gestores pela vinculação de cumprimento dos quatro componentes de avaliação listados na referida portaria como condição para habilitação.

A atenção básica assume assim um importante papel de co-partícipe na execução das políticas de saúde, como porta de entrada do sistema, como importante etapa no processo de regulação e como otimizadora das ações de saúde, por permitir a utilização racional dos recursos de maior complexidade.

A proposta de avaliação normativa contida na portaria 2.215, em que pese suas limitações, em especial, pelas dificuldades na coleta de dados para análise, trouxe à tona algumas discussões importantes.

Ocorreu um movimento de sistematização de informações de vários bancos de dados em nível nacional, relacionados direta ou indiretamente à atenção básica. Essa sistematização de dados, a transformação dos mesmos em informações e a divulgação dessas informações têm gerado ações efetivas, como a maior responsabilização dos municípios em relação à atenção básica. Vários municípios realizaram avaliações do processo de obtenção e registro de informações em nível local. Também neste quesito de registro de dados, foram observadas várias falhas relacionadas à subnotificação de procedimentos e ações de saúde, muitas vezes pela necessidade de se lançar o mesmo dado em diferentes sistemas nacionais de informação, ou mesmo, por falta de rotinas locais de registro, uma vez que os procedimentos de atenção básica não geram pagamento contra-produção.

Outro movimento desencadeado em muitos estados foi o de integração das áreas da atenção básica em busca de um melhor desempenho no processo global de acompanhamento, sendo fator estimulador inclusive de mudanças estruturais em algumas SES. Em vários estados, observou-se a aproximação de áreas de planejamento, controle e avaliação e de áreas técnicas de programas das coordenações de atenção básica.

A preocupação dos gestores municipais e estaduais com as metas a serem propostas no Pacto da Atenção Básica também é digna de nota. As mudanças no processo de pactuação, aliadas à avaliação de dois indicadores exigidos para habilitação, desencadearam discussões importantes sobre a responsabilidade dos gestores no processo de pactuação e corrigiram distorções importantes do processo. Algumas SES "pactuavam" metas fechadas, sem possibilidade de adequação por parte dos municípios. Quando havia liberdade para os municípios fixarem suas metas, observava-se, muitas vezes, que a meta fixada por eles não era factível, pois não havia passado por um processo de discussão mais aprofundado no próprio município.

\section{O controle e avaliação na Atenção Básica}

A implantação do Piso da Atenção Básica, com o repasse dos recursos do Fundo Nacional de Saúde diretamente aos Fundos Municipais de Saúde, modificou profundamente a lógica de financiamento da atenção básica. A posterior incorporação de outras ações através do PAB variável, que privilegiou projetos específicos como o PSF, PACS, Combate às Carências Nutricionais, entre outros, demonstrou um grande avanço na possibilidade de execução financeira por parte dos municípios, antes limitados às formas conveniais de repasses de recursos.

Entretanto, esses avanços não foram acompanhados da instituição de mecanismos de controle e avaliação, apesar do significativo volume de recursos repassados, para a execução das ações básicas de saúde. Em muitos casos, foram abandonadas as formas tradicionais de controle e avaliação, realizadas pelas áreas especificas das SES e Secretarias Municipais de Saúde (SMS), sem a substituição por outras propostas.

A atenção básica avançou na instituição da avaliação de processos e resultados, através do Pacto da Atenção Básica, instrumento com grande potencial de gestão.

A operacionalização da NOAS exige dos municípios habilitados em Gestão Plena da Atenção Básica Ampliada (GPAB-A) e Gestão Plena do Sistema Municipal (GPSM) a reavaliação dos seus Serviços de 
Controle e Avaliação para o cumprimento dos quesitos e responsabilidades inerentes à atenção básica. Essa é uma oportunidade de se repensar o processo de controle e avaliação da atenção básica, incorporando os avanços conquistados, de forma articulada com os serviços também existentes nas SMS e SES.

\section{Evolução do Programa Saúde da Família no Brasil}

A evolução do Programa Saúde da Família está relacionada ao processo de consolidação do Sistema Único de Saúde, respeitando as particularidades de cada região. Sua expansão demonstra importante adesão dos gestores municipais e estaduais, que compartilham responsabilidades com o Ministério da Saúde na busca da sustentabilidade do programa.

Se nos primeiros quatro anos de sua implantação, o PSF abrangia 3,51\% da população brasileira, ao final de 1998, este percentual atingiu 6,57\%. Em dezembro de 1999, existiam 4.114 equipes de Saúde da Família, atuando em 1.646 municípios, que representam 29,9\% dos municípios brasileiros. Eram aproximadamente 14 milhões de pessoas acompanhadas pelo programa, representando $8,8 \%$ da população do país. Em setembro de 2002, esses números cresceram significativamente: são 16.463 equipes, em 4.161 municípios, assistindo 54 milhões de pessoas.

A mudança do financiamento com a implantação do Piso da Atenção Básica e renovação das administrações municipais, respectivamente em 1997 e 2001, podem ter tido peso significativo para este crescimento.

O crescimento do PSF demonstra uma tendência inicial de cobertura de áreas menos assistidas, representadas por pequenos municípios, distantes dos aglomerados urbanos, e por cidades de médio porte. Esta tendência não ocorreu somente pela lógica natural de ampliar a cobertura em áreas desassistidas.

Aconteceu, neste período, importante incremen- to do acesso da população aos serviços de saúde, principalmente de segmentos da população expostos a maior vulnerabilidade social.

Porém, em 1999, quando da realização da Avaliação da Implantação e Funcionamento do Programa da Saúde da Família, identificou-se a tendência da pulverização de equipes de saúde da família nestes municípios, dificultando sua capacidade de reordenamento dos sistemas municipais de saúde, e conseqüentemente da reversão do modelo assistencial.

Para se disciplinar essa tendência, o Ministério da Saúde, a partir de novembro de 1999, além de reajustar valores, introduziu nova modalidade para o cálculo dos incentivos, passando a remunerar melhor os municípios que apresentam maior cobertura populacional pelas equipes de saúde da família.

Observou-se, assim, que, se em janeiro de 2000, $60 \%$ dos municípios possuíam apenas uma equipe de saúde da família e $3,13 \%$, mais de 10 . Atualmente, $6,42 \%$ dos municípios possuem mais de 10 equipes, enquanto $37,42 \%$ contam com apenas uma. É importante registrar o grande número de municípios que com apenas uma equipe de saúde da família cobrem toda a sua população.

A mudança na regra de financiamento mostrouse insuficiente para favorecer a ampliação do programa nas grandes cidades, onde os esforços, para ampliar a cobertura e, conseqüentemente, atingir faixas de maiores valores de incentivo, são muito grandes. Alguns entraves têm sido apontados pelos gestores. Entre eles, destacam-se a necessidade de se realizar um grande número de capacitação para os profissionais e a dificuldade de integração das equipes do PSF à rede de serviços já instalada.

Apesar dessas dificuldades, os municípios com mais de 100 mil habitantes assumiram a organização da atenção básica à saúde, segundo os princípios do PSF, como estratégia indispensável ao reordenamento do sistema de saúde. Este fato é constatado na evolução do programa nesses municípios, mostrada na Tabela 1. 
Evolução do Programa Saúde da Família em municípios com mais de 100 mil habitantes.

\begin{tabular}{|c|c|c|c|c|c|c|c|c|c|c|}
\hline \multirow{2}{*}{ Regiões } & \multicolumn{2}{|c|}{1998} & \multicolumn{2}{|c|}{1999} & \multicolumn{2}{|c|}{2000} & \multicolumn{2}{|c|}{2001} & \multicolumn{2}{|c|}{2002 (Setembro) } \\
\hline & $N^{\circ}$ ESF & $\%$ COB & $N^{\circ}$ ESF & $\% \mathrm{COB}$ & $N^{\circ}$ ESF & $\% \mathrm{COB}$ & $N^{\circ}$ ESF & $\% \mathrm{COB}$ & $N^{\circ}$ ESF & $\%$ COB \\
\hline Norte & 54 & 4,07 & 84 & 6,32 & 150 & 10,75 & 339 & 21,87 & 439 & 28,32 \\
\hline Nordeste & 363 & 7,66 & 540 & 11,39 & 875 & 17,84 & 1.213 & 23,56 & 1.558 & 30,26 \\
\hline Sul & 193 & 6,70 & 199 & 6,91 & 365 & 12,22 & 532 & 16,93 & 593 & 18,87 \\
\hline Centro-Oeste & 291 & 18,90 & 196 & 12,73 & 204 & 12,58 & 254 & 14,72 & 281 & 16,29 \\
\hline Total & 1.122 & 5,46 & 1.376 & 5,87 & 2.569 & 10,61 & 3.700 & 14,47 & 5.057 & 26,97 \\
\hline
\end{tabular}

ESF = Equipe de Saúde da Família $C O B=$ Cobertura populacional

Analisando-se a planilha, observa-se que houve um aumento de $350 \%$ e de $394 \%$, respectivamente, no número de equipes de Saúde da Família em atuação e na sua cobertura populacional, nesses quatro anos, nos municípios com mais de 100 mil habitantes. A região Sudeste apresenta o maior aumento percentual (889\%) no número de equipes implantadas e maior expansão da cobertura populacional, que foi de 2,19 para 15,62 , no período. Na região Centro-Oeste, ocorreu redução na implementação do programa, reflexo da desativação de um número significativo de equipes em Brasília, Distrito Federal, no início de 1999.

O PSF atende, hoje, $32,3 \%$ da população brasileira o que representa 55,7 milhões de pessoas. São 16.960 equipes atuando em todos os estados, numa abrangência de cobertura que chega a $75 \%$ das cidades do país.

O Ministério da Saúde tem como meta dobrar o número de equipes do Programa Saúde da Família nos próximos quatro anos, atingindo 100 milhões de habitantes, ou cerca de $77 \%$ da população brasileira. O projeto será efetivado com a capacitação de quatro a cinco mil equipes por ano em todo o país. Ao final de 2006 o PSF terá entre 30 a 32 mil equipes.

\section{O Ministério da Saúde vai destinar mais verbas para o PSF}

Hoje, os municípios recebem próximo de um terço dos recursos para pagamento das despesas do programa. A nova gestão da pasta da saúde pretende ainda debater com os gestores estaduais a possibilidade de construção de um pacto entre os administradores das três esferas do governo (municipal, estadual e federal) para que seja estabelecida uma divisão de incentivos para funcionamento do PSF em cada município. A intenção é que os governos estaduais contribuam com $25 \%$ dos recursos financeiros, as prefeituras municipais com $25 \%$ cabendo ao Mi-nistério os $50 \%$ restantes.

Essas novas metas do PSF serão efetivadas com o apoio do Projeto de Expansão e Consolidação do Saúde da Família (PROESF). O acordo, selado entre o governo federal e o Banco Mundial, colocará à disposição do Programa US\$550 milhões em sete anos para serem utilizados em atividades que envolvam a manutenção da saúde da família. Desse valor, metade será financiada pelo BIRD e a outra metade representa a contrapartida do governo brasileiro.

Recebido em 6 de Janeiro de 2003

Versão final reapresentada 10 de Janeiro de 2003

Aprovada em 14 de Janeiro de 2003 